\title{
Prediction of plasma leakage phase of dengue in resource limited settings
}

\author{
H.M.M.T.B. Herath ${ }^{\mathrm{a}, *}$, W.A.E. Udeshika ${ }^{\mathrm{a}}$, S.S.M. Samarawickrama ${ }^{\mathrm{a}}$, \\ Nilukshana Yogendranathan $^{\mathrm{a}}$, W.D. Jayamali ${ }^{\mathrm{a}}$, Aruna Kulatunga ${ }^{\mathrm{a}}$, Chaturaka Rodrigo ${ }^{\mathrm{b}, \mathrm{c}}$ \\ a National Hospital, Colombo, Sri Lanka \\ ${ }^{\mathrm{b}}$ Department of Clinical Medicine, Faculty of Medicine, University of Colombo, Sri Lanka \\ ${ }^{\mathrm{c}}$ Department of Pathology, School of Medical Sciences, University of New South Wales, Sydney, 2052, Australia
}

\section{A R T I C L E I N F O}

\section{Keywords:}

Dengue

Plasma leakage

Risk prediction

Liver tenderness

\begin{abstract}
A B S T R A C T
Introduction: The pathophysiology of severe dengue is related to increased capillary permeability and plasma leakage into extracellular space. A simple, low cost risk prediction tool for plasma leakage will be useful for clinicians practicing in rural areas without imaging facilities.

Study design: A prospective observational study was carried out over 12 months at the National Hospital, Sri Lanka enrolling patients with confirmed diagnosis (via NS1 antigen testing) of early dengue infection. Clinical features on admission and investigation results on D3, D5 and D7 of the illness were recorded. Evidence of plasma leakage was confirmed by ultrasonography.

Results: A total of 179 patients met the inclusion criteria (males; 91, 50.8\%, mean age: 31.6 years, SD \pm 14.7 ). Sixty seven patients $(67 / 173,38.7 \%)$ had ultrasonographic evidence of plasma leakage. Several clinical features (severe vomiting, severe diarrhoea, abdominal pain and liver tenderness) as well as mean differences of some investigations were significantly associated with progression to plasma leakage. However, only liver tenderness on day 3 emerged as independent significant predictors of critical phase in the adjusted analysis (specificity: 93\%, sensitivity: 44\%).

Conclusions: Having liver tenderness by day 3 of the illness is helpful to identify a subgroup of patients at risk of plasma leakage.
\end{abstract}

\section{Introduction}

Incidence of dengue fever has increased globally over the last two decades from an estimated 8.3 million cases in 1990 to 58.3 million in 2013. ${ }^{1}$ The total disability adjusted life years lost due to dengue was 1.14 million in $2013 .{ }^{1}$ In the first seven months of 2017, more than 100,000 confirmed dengue cases were reported in Sri Lanka resulting in over 300 deaths with a case fatality rate of $0.2-0.3 \% .^{2}$

Clinically, manifestations of dengue fever vary from an asymptomatic illness to the potentially lethal dengue shock syndrome. A majority of symptomatic patients will only have a flu-like illness ${ }^{3}$ and a relatively smaller proportion of these patients will enter a "critical phase" characterized by increased capillary permeability and loss of intravascular fluid to the extravascular compartment. ${ }^{3}$ Repeated observational studies suggest that the plasma leakage is short lasting for approximately $48 \mathrm{~h}^{4}$ Close supervision and management is essential during this period as hypovolaemia due to undetected loss of intravascular volume can lead to shock, organ dysfunction and death. ${ }^{3}$ Similarly, over enthusiastic fluid administration during this time can result in pulmonary oedema and fluid overload.

Detection of this short lasting phase of plasma leakage is a challenge in resource-limited settings. The most reliable way to do this is to conduct frequent ultrasound scans to demonstrate fluid accumulation in the pleural or peritoneal cavities. Clinical examination is not sensitive enough to pick up small fluid accumulations and chest radiographs will not show a pleural effusion until $400-500 \mathrm{ml}$ of fluid has accumulated. Attempts to identify the leakage phase by monitoring haematological parameters such as the haematocrit (packed cell volume) can be unreliable unless assessment is repeated every few hours. A hallmark of dengue fever is the transient thrombocytopaenia and many clinicians consider thrombocytopaenia (a dip in platelet count to less than $100,000 / \mu \mathrm{l})$ to precede the onset of plasma leakage by $24-36 \mathrm{~h}$. Recommendations based on this observation have been incorporated in to guidelines to predict critical phase. ${ }^{4}$

\footnotetext{
* Corresponding author.

E-mail addresses: tharukaherath11@gmail.com (H.M.M.T.B. Herath), Waeudeshika@gmail.com (W.A.E. Udeshika), sincysms@gmail.com (S.S.M. Samarawickrama),ynilu6@gmail.com (N. Yogendranathan), danurulzzz@yahoo.com (W.D. Jayamali), arunakulatunga@yahoo.com (A. Kulatunga), c.rodrigo@unsw.edu.au (C. Rodrigo).
} 
Due to financial constraints it is usually not possible to confirm a diagnosis of dengue serologically or to confirm fluid leakage by ultrasound. During the dengue epidemic in Sri Lanka in 2017, a large number of patients in various stages of dengue fever were admitted to the National Hospital of Sri Lanka, the premier state sector, tertiary care referral centre in the country. Due to facilities available in this hospital it was possible to serologically confirm a diagnosis of dengue and to confirm the onset of leakage phase via frequent ultrasonography thus overcoming two major obstacles in clinical research on dengue leakage phase. This study aims to identify the value of clinical features and investigation findings in early dengue infection to predict the subsequent occurrence of plasma leakage.

\section{Material and methods}

This prospective, hospital based cross-sectional, observational study was conducted in two of the eight general medical wards of the National Hospital of Sri Lanka (NHSL). All patients to the medical wards are admitted randomly in rotation by an independent admitting medical officer at the outpatients department. As mentioned previously, the National Hospital of Sri Lanka is the premier public health care centre and the largest tertiary care referral centre in the country. It is located in the Western Province of Sri Lanka which has the highest incidence of dengue in the country. ${ }^{2}$

All consenting adult patients with a diagnosis of dengue as confirmed by an NS1 (non structural region 1) antigen test and admitted for in-ward management of the illness were included. NS 1 antigen test has a high sensitivity and specificity in early dengue infection up to day 4 of the illness. ${ }^{5}$ The method of detection was through enzyme-linked immunosorbent assay and all patients with suspicion of dengue fever (acute onset of fever with headache, especially retro-orbital pain, myalgia/arthralgia, rash, haemorrhagic manifestations, leukopenia of $<5000 / \mathrm{mm}$, rising haematocrit of $5-10 \%$ and platelet count $\leq 150,000 / \mathrm{mm}^{3}$ ) underwent NS1 testing. ${ }^{4}$

Exclusion criteria were as follows; a) non-consenting patients, b) patients already discovered to have plasma leakage or in recovery stage at admission, c) patients without a confirmed diagnosis by NS 1 antigen testing. A selected set of clinical features and investigation results (determined a priori) of included patients were recorded (see below). The days of fever were counted by taking the first day of fever as day 1 (D1). When the patient already had fever on admission, D1 was determined from the clinical history. All investigations were done according to the dengue management guidelines ${ }^{4}$ by medical officers under the supervision of the attending consultant. None of the investigations were done for the study per se as this was an observational study. Most investigations included in this study can be done at a resource limited peripheral hospital in Sri Lanka. These tests were done at D3, D5 and D7 of the illness and included; full blood count, serum alanine aminotransferase (ALT), serum aspartate aminotransferase (AST), serum proteins, C-reactive protein and serum ionized calcium. Two derivatives of the raw data (ALT/AST ratio, neutrophil/lymphocyte ratio) were also recorded. Follow up data were not collected after discharge from the hospital.

The presence or absence of plasma leakage (primary outcome) during the illness was confirmed by ultrasonographic evidence of fluid leakage. Ultrasonography was carried out a) when the platelet count dropped below $100,000 / \mu 1$ or b) when serial haematocrit monitoring showed haemoconcentration more than $20 \%$ of the baseline value or c) when there was clinical evidence of pleural effusion or ascites. If the initial scan did not show extravasated fluid, the scan was repeated every 8-12 h until fluid leakage was confirmed or the clinical condition improved.

Clinical features to be correlated with the presence or absence of leakage were decided a priori. The clinical features selected included severe diarrhoea (more than three times a day), severe vomiting (more than 3 times a day), liver tenderness on palpation and abdominal pain.
Presence of these clinical symptoms was recorded if they were present at any time prior to the onset of plasma leakage.

The statistical analysis was done with SPSS (version 20, IBM, USA) statistical package. The descriptive statistics were summarized as sum of counts for categorical variables and as mean and standard deviation for continuous variables. Significant associations for the presence of plasma leakage were evaluated with chi square test for categorical variables and using mean differences for continuous variables (independent $\mathrm{T}$ test). Any significant findings on univariate analysis were further assessed by logistic regression. Ethical clearance for the study was obtained from the ethics review committee of the National Hospital of Sri Lanka.

\section{Results}

This study was conducted over a period of 12 months from August 2016 to August 2017. NHSL does not admit paediatric patients (under 12 years of age) to its medical wards. One hundred and seventy nine (n179) patients met the inclusion criteria (males; $91,50.8 \%$, mean age: 31.6 years, $S D \pm 14.7)$. The mean number of days with fever on admission was 3.36 days $(S D \pm 1.12)$ and the total duration of fever was 4.51 days (SD \pm 1.14 ) for the study cohort. Sixty seven patients (67/ $173,38.7 \%$ ) had ultrasonographic evidence of plasma leakage during the course of the illness and the mean estimate for the onset of plasma leakage was 5.04 days ( $S D \pm 1.04)$ since the onset of fever. In this group, the platelet count dropped below $100,000 / \mu \mathrm{l}$ at 3.42 days $(\mathrm{SD} \pm 1.6)$. All patients recovered fully and were discharged from the hospital. A detailed description of the clinical parameters of the study sample is given in Table 1 .

On univariate analysis all clinical features (vomiting, diarrhoea, abdominal pain, liver tenderness) were significantly associated with subsequent progression to plasma leakage (Table 2). However after logistic regression only liver tenderness showed a significant association with plasma leakage. Regarding the investigation results at D3, only a lower platelet count had a significant association with progression to plasma leakage phase $\left(94.84 \times 10^{3} / \mu 1\right.$ vs. $123.95 \times 10^{3} / \mu 1$, $\mathrm{p}<0.001$ ). However, as the selection criteria for ultrasonography included thrombocytopaenia, this result is biased was not analysed further. By D5, the total mean WBC count, mean haematocrit, aspartate aminotransferase (AST) and alanine aminotransferase (ALT) were significantly higher in patients that had plasma leakage $(\mathrm{p}<0.05$, Table 3). Regarding the D7 investigation results, the total mean WBC count and AST level were significantly higher in patients with plasma leakage $(\mathrm{p}<0.05)$ while the mean platelet count, ALT/AST ratio and the serum albumin level were significantly lower $(\mathrm{p}<0.05)$. After a logistic regression, none of these parameters remained as significant

Table 1

Descriptive statistics of the clinical variables of the sample $(\mathrm{N}-179)^{\mathrm{a}}$.

\begin{tabular}{|c|c|c|}
\hline Variable & Mean $( \pm S D)$ & Number (\%) \\
\hline Age & $31.6(14.7)$ & \\
\hline \multicolumn{3}{|l|}{ Sex } \\
\hline Male & & $91(50.8)$ \\
\hline Female & & $88(49.2)$ \\
\hline Number of days of fever at presentation (n-176) & $3.36(1.1)$ & \\
\hline Number of patients entering critical phase & & $67(38.7)$ \\
\hline $\begin{array}{l}\text { The day of having the first platelet count below } \\
100,000 / \mu 1^{* *}\end{array}$ & $3.42(1.6)$ & \\
\hline The day of entering critical phase ${ }^{* *}$ & $5.04(1.0)$ & \\
\hline Total number of days with fever (n-170) & $4.51(1.1)$ & \\
\hline \multicolumn{3}{|l|}{ Clinical features } \\
\hline Severe vomiting ( $>3 /$ day, $n-166$ ) & & $76(45.8)$ \\
\hline Severe diarrhoea ( $>3 /$ day, $n-165$ ) & & $36(21.8)$ \\
\hline Abdominal pain $(n-164)$ & & $39(23.8)$ \\
\hline Liver tenderness ( $n-161)$ & & $36(22.4)$ \\
\hline
\end{tabular}

a The first day of fever is taken as day $1,{ }^{*}$ *only for patients that entered the critical phase (n-67). 
Table 2

Clinical predictors of having a critical phase.

\begin{tabular}{lllll}
\hline Variable $^{\mathrm{a}}$ & $\begin{array}{l}\text { Unadjusted odd } \\
\text { ratio }( \pm 95 \% \mathrm{CI})\end{array}$ & P value & $\begin{array}{l}\text { Adjusted odd ratio } \\
( \pm 95 \% \mathrm{CI})\end{array}$ & P value \\
\hline Severe vomiting & $3.51(1.81-6.82)$ & $<0.001$ & & \\
Severe diarrhoea & $2.71(1.24-5.94)$ & 0.015 & & \\
Abdominal pain & $\begin{array}{l}\text { 4.55(2.07-10.01) } \\
<0.001\end{array}$ & & & \\
Liver tenderness & $9.87(3.93-24.79)$ & $<0.001$ & $7.87(2.58-24.39)$ & $<0.001$ \\
\hline
\end{tabular}

a Odds are compared for those with the clinical feature vs. others and only shown for statistically significant associations.

Table 3

Investigation results with significant associations for critical phase (CP) as assessed by mean difference*.

\begin{tabular}{|c|c|c|c|}
\hline \multirow[t]{2}{*}{ Variable* } & \multicolumn{2}{|l|}{ Mean values $( \pm S D)$} & \multirow[t]{2}{*}{$P$ value } \\
\hline & Patients with $C P$ & Patients without $C P$ & \\
\hline D5 white cell count & $4.19 \times 10^{3} / \mu \mathrm{l}(2.72)$ & $3.34 \times 10^{3} / \mu 1(1.81)$ & 0.029 \\
\hline D5 haematocrit & $41.11 \%(5.44)$ & $39.39 \%(4.17)$ & 0.037 \\
\hline D5 AST*** & $361.46 \mathrm{IU} / 1(770.27)$ & $108.71 \mathrm{IU} / 1(97.62)$ & 0.020 \\
\hline D5 $\mathrm{ALT}^{* * *}$ & $220.46 \mathrm{IU} / 1$ (356.69) & 80.09 IU/1 (74.98) & 0.007 \\
\hline D7 white cell count & $6.78 \times 10^{3} / \mu \mathrm{l}(3.53)$ & $5.15 \times 10^{3} / \mu \mathrm{l}(2.71)$ & 0.002 \\
\hline D7 AST & $274.92 \mathrm{IU} / 1$ (439.72) & 109.16 IU/1 (113.44) & 0.031 \\
\hline D7 serum albumin & $32.77 \mathrm{~g} / 1$ (5.69) & $36.53 \mathrm{~g} / \mathrm{l}(3.18)$ & 0.038 \\
\hline
\end{tabular}

*Only statistically significant associations are shown, platelet count at D3, D5 and D7 all showed a significantly low value in those with leakage. However as the selection criteria for ultrasonography included platelet count this observation is biased and hence not mentioned, ** First day of fever is counted as day 1 (D1), $* * *$ AST - aspartate aminotransferase, ALT - alanine aminotransferase measured in international units per litre (IU/1).

predictors for having plasma leakage.

Overall, liver tenderness had 93\% (95\% CI: 85-97\%) specificity and 44\% (95\% CI: 32-58\%) sensitivity in predicting plasma leakage. The positive and negative predictive values were 79\% (95\% CI: $64-89 \%$ ) and $72 \%$ (95\% CI: $67-76 \%)$ respectively.

\section{Discussion}

In this prospective observational study abdominal pain and liver tenderness were selected as previous authors have identified hepatic inflammation to be associated with severe dengue infections ${ }^{6-8}$ Vomiting and diarrhoea were selected as it may exacerbate intravascular hypovolaemia leading to an adverse outcome. Demographic factors (ethnicity, level of income, educational level) other than age and gender were not considered as the influence of these factors would vary in different communities limiting their usefulness in a generalized risk prediction algorithm. In this study liver tenderness in early infection was independently and significantly associated with plasma leakage during the illness. While this was not sensitive enough to identify all patients with leakage, in resource limited settings where ultrasonography is not available, it may be useful to identify a subgroup of patients more likely to have plasma leakage. Unfortunately not having liver tenderness does not guarantee patient safety.

Dengue hepatitis is a relatively uncommon but a well recognized complication of the infection. ${ }^{6}$ Asymptomatic elevation of serum aminotransferase levels of hepatic origin is more common and has been associated with lower platelet counts previously in a large cohort of patients in India (n- 327). ${ }^{9}$ However, the authors had not assessed its relationship to the onset of the plasma leakage which is an important outcome when it comes to patient management. The liver enzymes may continue to rise after the critical phase and may even peak during the convalescent phase. ${ }^{7,8,10}$ Several other studies (both prospective and retrospective) have found a more direct link between elevated liver enzymes and severe disease as defined by outcomes such as bleeding, mortality and increased hospital stay both in adult and paediatric patients. $^{8,} 11-13$ Almost all complications of dengue including bleeding and death occur in patients who have plasma leakage and therefore it would be more useful to seek predictors of leakage rather than that of complications for purposes of prevention of the latter. In this study we demonstrate that liver enzymes per se were not useful as predictors but clinical evidence of liver tenderness to palpation was a strong and independent predictor of impending leakage phase.

Current guidelines in dengue management suggest that a $20 \%$ rise in haematocrit or a drop in platelet count below $100,000 / \mu 1$ are likely to precede the onset of critical phase by $24-48 \mathrm{~h} .{ }^{4}$ There are many studies that provide observational data to confirm these recommendations. ${ }^{14-17}$ In our analysis, a rise in haematocrit at D3 was significant on univariate analysis but it was not an independent predictor on regression analysis. This finding does not underestimate the usefulness of haematocrit measurements. Changes in haematocrit are very dynamic and are often used to confirm the plasma leakage when it has occurred (unless there is concurrent bleeding). At least 6-8 hourly haematocrit assessments should be done in high risk patients to pick up the onset of leakage phase when ultrasonography is not available. However, because of these very dynamic fluctuations it may not be useful as a predictive indicator up to $36 \mathrm{~h}$ prior to the onset of leakage. In this regard platelet count was observed to be a more robust and early indicator than the more dynamic haematocrit measurements.

Risk prediction algorithms in a clinical setting have value only if they are simple, quick and inexpensive to be applied on a mass scale at times of epidemics even in rural hospitals without imaging facilities. In this regard, expensive investigations such as interleukin -10 , soluble cell adhesion molecules, thrombomodulin or even dengue serotyping are not useful despite being potential predictors of plasma leakage. ${ }^{15,18,19}$ Several authors have discussed the role of demographic factors (ethnicity, income and education) and co-morbidities (diabetes and hypertension) in predicting leakage phase. ${ }^{20}$ Given that dengue is an acute illness, it is very difficult to differentiate between spurious statistical associations and true associations when too many variables are assessed. As mentioned previously including demographic variables could have affected the generalizability of a risk prediction algorithm. Therefore we avoided demographic variables (other than gender and age) in our a priori selection and only included easily assessable, pathophysiologically relevant clinical features and investigation results. Though some studies have found older age to be a significant predictor of disease severity, ${ }^{21}$ we did not observe such an association probably as our cohort was a relatively younger with a mean age of 31.6 years (SD \pm 14.7 years). Similar risk prediction algorithms based on WBC counts and platelet levels (for paediatric patients) ${ }^{22}$ or history of bleeding, blood urea and total protein levels (by a retrospective analysis $)^{23}$ have been published previously to predict dengue shock syndrome. While both algorithms had high specificity (97-100\%) the sensitivity was low (46-48\%).

\section{Limitations}

This study only included patients above 12 years of age. The ultrasonography carried out during the time of epidemic was a focussed limited sonographic examination subject to inter-observer bias. However, all scans were performed by trained medical officers in the radiology department. Similarly there is a potential inter-observer bias in detecting liver tenderness. All medical officers involved in the study were well trained on clinical examination skills. Due to limited resources we could not offer ultrasonography to all patients. Instead, patients were screened based on thrombocytopaenia to observe a higher yield in detecting plasma leakage. Though the final results showed an association with thrombocytopaenia and plasma leakage in the analysis, this was not included as a result due to the selection bias.

The only outcome defined in this study is the presence or absence of plasma leakage. Data on disease severity or follow up data were not 
collected. Including body mass index as a potential predictor would have been useful as this can be an indirect indicator of hepatic steatosis. Unfortunately this was not included in the initial study protocol.

\section{Conclusions}

An assessment of 179 patients with confirmed early dengue infection showed that having severe vomiting, diarrhoea, abdominal pain and liver tenderness was significantly associated with progression to plasma leakage during the illness. Similarly there were significant mean differences of AST, ALT, WBC and haematocrit values on D5; AST, WBC and serum albumin level on D7 in patients with plasma leakage compared to others. However, only liver tenderness on D3 emerged as an independent significant predictor for plasma leakage in the adjusted analysis. This will be useful for clinicians working in resource limited peripheral hospitals without imaging facilities to identify a subset of patients with a high likelihood of having plasma leakage.

\section{List of abbreviations}

AST: aspartate aminotransferase, ALT: alanine aminotransferase, CP: Critical phase, NHSL: National Hospital of Sri Lanka, NS1: Non structural region 1, WBC: While blood cell

\section{Declarations}

\section{Ethical approval and consent to participate}

This was obtained from the ethics review committee of National Hospital of Sri Lanka. Informed written consent was obtained from patients and parents or guardians on behalf of any participants under the age of 16 , prior to data collection. Personal information was deidentified prior to recording.

\section{Consent for publication}

Not relevant as patient data is de-identified.

\section{Availability of data and material}

The data set for this publication is available upon request from the authors.

\section{Conflicts of interest}

None to declare.

Funding

None.

\section{Author contributions}

HMMTBH and CR conceptualized the study. HMMTBH WAEU SSMS NY WDJ collected the data. AK was responsible for clinical care of patients. AK and CR supervised the project. CR and HMMTBH wrote the first draft. All authors revised and approved the final manuscript.

\section{Acknowledgements}

None.

\section{References}

1. Stanaway JD, Shepard DS, Undurraga EA, et al. The global burden of dengue: an analysis from the Global Burden of Disease Study 2013. Lancet Infect Dis. 2016;16(6):712-723.

2. Dengue Update [http://www.epid.gov.lk/web/index.php?option $=$ com_content\& view $=$ article\&id $=171 \% 3$ Adengue-update $\&$ catid $=51 \% 3$ Amessage-for-public $\&$ Itemid $=487 \&$ lang $=$ en $]$.

3. Rajapakse S, Rodrigo C, Rajapakse A. Treatment of dengue fever. Infect Drug Resist. 2012;5:103-112.

4. Ministry of Health Sri Lanka: Guidelines on Management of Dengue Fever and Dengue Haemorrhagic Fever in Adults. Colombo, Sri Lanka: Ministry of Health Sri Lanka; 2012.

5. Pal S, Dauner AL, Mitra I, et al. Evaluation of dengue NS1 antigen rapid tests and ELISA kits using clinical samples. PloS One. 2014;9(11):e113411.

6. Gulati S, Maheshwari A. Atypical manifestations of dengue. Trop Med Int Health: TM \& IH. 2007;12(9):1087-1095.

7. Parkash O, Almas A, Jafri SM, Hamid S, Akhtar J, Alishah H. Severity of acute hepatitis and its outcome in patients with dengue fever in a tertiary care hospital Karachi, Pakistan (South Asia). BMC Gastroenterol. 2010;10:43.

8. Trung DT, Thao le TT, Hien TT, et al. Liver involvement associated with dengue infection in adults in Vietnam. Am J Trop Med Hyg. 2010;83(4):774-780.

9. Ahmad S, Dhar M, Srivastava S, et al. Dengue hepatitis sans dysfunction: experience of a single tertiary referral centre in the north Indian state of Uttarakhand. Trop Doct. 2013;43(2):62-65.

10. Kuo CH, Tai DI, Chang-Chien CS, Lan CK, Chiou SS, Liaw YF. Liver biochemical tests and dengue fever. Am J Trop Med Hyg. 1992;47(3):265-270.

11. Wichmann O, Gascon J, Schunk M, et al. Severe dengue virus infection in travelers: risk factors and laboratory indicators. J Infect Dis. 2007;195(8):1089-1096.

12. Kalayanarooj S, Vaughn DW, Nimmannitya S, et al. Early clinical and laboratory indicators of acute dengue illness. J Infect Dis. 1997;176(2):313-321.

13. Lee LK, Gan VC, Lee VJ, Tan AS, Leo YS, Lye DC. Clinical relevance and discriminatory value of elevated liver aminotransferase levels for dengue severity. PLoS Neglected Trop Dis. 2012;6(6):e1676.

14. Potts JA, Rothman AL. Clinical and laboratory features that distinguish dengue from other febrile illnesses in endemic populations. Trop Med Int Health: TM \& IH. 2008;13(11):1328-1340.

15. Brasier AR, Ju H, Garcia J, et al. A three-component biomarker panel for prediction of dengue hemorrhagic fever. Am J Trop Med Hyg. 2012;86(2):341-348.

16. Krishnamurti C, Kalayanarooj S, Cutting MA, et al. Mechanisms of hemorrhage in dengue without circulatory collapse. Am J Trop Med Hyg. 2001;65(6):840-847.

17. Wills B, Tran VN, Nguyen TH, et al. Hemostatic changes in Vietnamese children with mild dengue correlate with the severity of vascular leakage rather than bleeding. Am J Trop Med Hyg. 2009;81(4):638-644.

18. Cardier JE, Rivas B, Romano E, et al. Evidence of vascular damage in dengue disease: demonstration of high levels of soluble cell adhesion molecules and circulating endothelial cells. Endothelium: J. Endothelial Cell Res. 2006;13(5):335-340.

19. Butthep P, Chunhakan S, Tangnararatchakit K, Yoksan S, Pattanapanyasat K, Chuansumrit A. Elevated soluble thrombomodulin in the febrile stage related to patients at risk for dengue shock syndrome. Pediatr Infect Dis $J$. 2006;25(10):894-897.

20. Figueiredo MA, Rodrigues LC, Barreto ML, et al. Allergies and diabetes as risk factors for dengue hemorrhagic fever: results of a case control study. PLoS Neglected Trop Dis. 2010;4(6):e699.

21. Karunakaran A, Ilyas WM, Sheen SF, Jose NK, Nujum ZT. Risk factors of mortality among dengue patients admitted to a tertiary care setting in Kerala, India. J. Infection Publ. Health. 2014;7(2):114-120.

22. Potts JA, Gibbons RV, Rothman AL, et al. Prediction of dengue disease severity among pediatric Thai patients using early clinical laboratory indicators. PLoS Neglected Trop Dis. 2010;4(8):e769.

23. Lee VJ, Lye DC, Sun Y, Leo YS. Decision tree algorithm in deciding hospitalization for adult patients with dengue haemorrhagic fever in Singapore. Trop Med Int Health: TM \& IH. 2009;14(9):1154-1159 